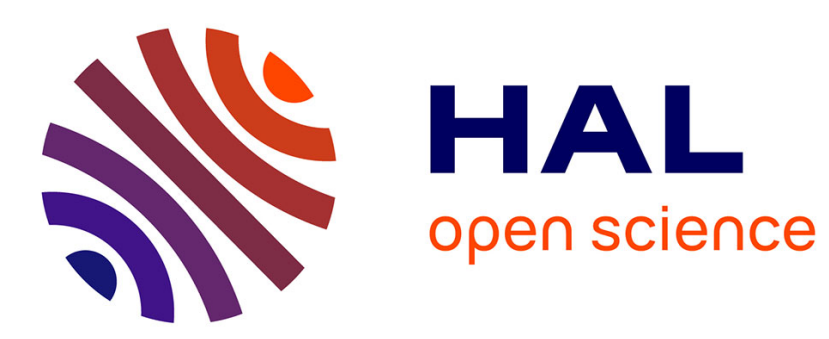

\title{
Alpha effect in helically forced turbulence
}

Olivier Agullo, Sadruddin Benkadda

\section{To cite this version:}

Olivier Agullo, Sadruddin Benkadda. Alpha effect in helically forced turbulence. Communications in Nonlinear Science and Numerical Simulation, 2003, 8 (3-4), pp.455 - 467. 10.1016/S10075704(03)00026-1 . hal-01625878

\section{HAL Id: hal-01625878 \\ https://hal-amu.archives-ouvertes.fr/hal-01625878}

Submitted on 29 Oct 2017

HAL is a multi-disciplinary open access archive for the deposit and dissemination of scientific research documents, whether they are published or not. The documents may come from teaching and research institutions in France or abroad, or from public or private research centers.
L'archive ouverte pluridisciplinaire HAL, est destinée au dépôt et à la diffusion de documents scientifiques de niveau recherche, publiés ou non, émanant des établissements d'enseignement et de recherche français ou étrangers, des laboratoires publics ou privés. 


\title{
Alpha effect in helically forced turbulence
}

\author{
Olivier Agullo *, Sadruddin Benkadda \\ LPIIM, CNRS/Université de Provence, Centre universitaire de Saint-Jérôme, F-13397 Marseille Cedex 20, France
}

\begin{abstract}
The effect on large-scale dynamics of small-scale helicity injection in three-dimensional resistive magnetohydrodynamic turbulence is investigated for a weak initial magnetic field. The magnetic configuration of $\alpha^{2}$ dynamo prototype flows is such that, generally, the energy concentrates on one large-scale mode. However, we obtain that alpha effect is not limited to the dominant mode and that a non-local equation (in Fourier space) is more appropriate to describe it. It gives some insights into the non-local theory of Pouquet et al. [J. Fluid Mech. 77 (1976) 321] where the inverse cascade results from a competition between the helicity and Alfvén effects.
\end{abstract}

(C) 2003 Elsevier B.V. All rights reserved.

Keywords: Dynamo; Plasma turbulence; MHD; Alpha effect; Helical forcing

\section{Introduction}

The origin of cosmic magnetic fields is often explained by dynamo theory, a branch of magnetohydrodynamics (MHD) regarding the self-excitation of a magnetic field by plasma or fluid motions [2]. To describe the generation of large-scale magnetic fields from small-scale turbulence some mechanisms have been proposed and, in particular, many works focused on $\alpha^{2}$ and $\alpha-\omega$ dynamos [3,4]. However MHD turbulence can not be reduced to these mechanisms and, for instance, the combination of Alfvén effect and the inverse cascade process (which can be, eventually, linked to an alpha mechanism) of magnetic helicity can also stimulate an important non-linear turbulent dynamo process [1,5]. In fact, Alfvén effect, i.e. generation of Alvén waves, can promote a rapid equipartition of magnetic and kinetic energy and, as a consequence, $\alpha$ effect (if any) is reduced. However, on the one hand, for Alfvén effect to be very efficient, we need an initial

\footnotetext{
* Corresponding author.

E-mail address: agullo@up.univ-mrs.fr (O. Agullo).
} 
large-scale magnetic field (ideally with a non-zero mean) and a strong turbulence. The former point is not necessary in the case of astrophysical problems. On the other hand, to study the dynamo problem, it can be interesting to focus on simplified systems where not all the mechanisms are fully involved. In particular, in this paper, there is no net rotation of the flow and the mean magnetic field is null. As a consequence, Alvén effect is not directly involved in the amplification mechanism of the largest scales. In fact, the system develops self-consistently a sheared magnetic field at moderate Reynolds numbers allowing to get some insight about $\alpha$ effect.

Cattaneo et al. [6] presented in the frame of the resistive MHD, numerical results showing that alpha effect is not the underlying mechanism of dynamo effect at high magnetic Reynolds number $R_{\mathrm{m}}$. They showed in fact that it decreases as the inverse of $R_{\mathrm{m}}$, in agreement with the theoretical predictions of Gruzinov et al. [7]. Blackman et al. [8] suggested, using analytic developments, that this result is strongly correlated to the combination of periodic boundary conditions and the existence of an uniform mean field in the simulation. In fact, the existence (or not) of the quenching of the alpha effect is not clear and might depend strongly on the global geometry of the system studied $[9,10]$. However, all these studies have in common that they study an alpha effect based on an hypothesis of scale separation, a so-called two-scale turbulence (see for instance [5] for a derivation of the theory). Even if some justifications to such an hypothesis can be given (for instance, for the earth see [3]), scale separation is not, à priori, the dominant character of all the astrophysical bodies. In addition, in their pioneering work Pouquet et al. [1], using an Eddydamped quasi-normal Markovian approximation, obtained analytically a non-local alpha effect (in some limits the usual alpha effect is recovered). Surprisingly, little attention has been devoted to this result. In the next section we start by presenting a general study of the effect of helicity in a turbulent flow. We then focus on the study of helical waves at the largest scales and discuss the relation to a non-local alpha effect.

\section{Effect of helicity in turbulent flows}

The main theoretical model of alpha-dynamo generation specifies that a large-scale magnetic field $\mathbf{b}_{\mathrm{LS}}$ evolves, as a first approximation, according to the model equation

$$
\frac{\partial}{\partial t} \mathbf{b}_{\mathrm{LS}}=\alpha \nabla \times \mathbf{b}_{\mathrm{LS}}+\beta \Delta \mathbf{b}_{\mathrm{LS}},
$$

where $\alpha$ and $\beta$ are parameters controlled by turbulent and statistical properties of the velocity field. Indeed, kinematic approaches predict that $\alpha$ and $\beta$ are respectively functions of the fluid helicity spectrum and the kinematic spectrum [3]. In particular $\alpha$ effect is associated to helical flows. Before studying it, we start by emphasizing the difference between helical and non-helical flows. To do it, we present simulations of the resistive MHD equations.

Let us introduce some definitions. The energy, helicity and relative helicity, for a given field $\mathbf{g}$, are

$$
E(\mathbf{g})=\frac{1}{2} \int_{\mathscr{D}} \mathrm{d}^{3} \mathbf{r g}^{2},
$$




$$
\begin{aligned}
& H(\mathbf{g})=\int_{\mathscr{D}} \mathrm{d}^{3} \mathbf{r g} \cdot \nabla \times \mathbf{g}, \\
& H_{r}(\mathbf{g})=H(\mathbf{g}) /[E(\mathbf{g}) E(\nabla \times \mathbf{g})] .
\end{aligned}
$$

$\mathscr{D}$ denotes the three-dimensional periodic space and by construction $\left|H_{r}(\mathbf{g})\right| \leqslant+1$. The equations of incompressible MHD, written in the usual units, read

$$
\begin{aligned}
& \frac{\mathrm{D}}{\mathrm{D} t} \mathbf{u}=-\nabla p+(\mathbf{b} \cdot \nabla) \mathbf{b}+v \nabla^{2} \mathbf{u}+\mathbf{f}, \\
& \frac{\mathrm{D}}{\mathrm{D} t} \mathbf{b}=\mathbf{b} \cdot \nabla \mathbf{u}+\eta \nabla^{2} \mathbf{b}, \\
& \nabla \cdot \mathbf{b}=\nabla \cdot \mathbf{u}=0,
\end{aligned}
$$

where, by definition, $\eta$ is the magnetic diffusivity, $v$ the kinematic viscosity and the pressure term $p$ is the sum of the hydrostatic pressure and the magnetic pressure. Here, both fluid velocity $\mathbf{u}$ and magnetic field $\mathbf{b}$ are expressed in Alfvèn speed units. $E(\mathbf{u})+E(\mathbf{g}), H(\mathbf{a})$ and $\int_{D} \mathrm{~d}^{3} \mathbf{r u} \cdot \mathbf{b}$, respectively the total energy, the magnetic helicity and the cross helicity, are the ideal quadratic invariants of the MHD equations. $\mathbf{a}$ is the magnetic potential vector $(\mathbf{b}=\nabla \times \mathbf{a})$. The magnetic Prandtl number is $P_{\mathrm{m}}=\eta / v$ and we restrict ourselves to $P_{\mathrm{m}}=1$.

The flow is forced by divergence free fields $\mathbf{f}$ centered around $k=5$ with random phases. One could choose to force at much smaller scales in order to be coherent with two-scales turbulence hypothesis leading to Eq. (1). However, we checked that numerical results concerned by alpha effect are not sensitive to it. A few modes are updated randomly at every time step in order to decorrelate the forcing in time. Moreover, in the following, we will analyze the effect of two kinds of forcing named forcing $A$ and forcing $B$. Let us emphasize that in both cases the forcings have the same spectrum but not the same phases. Constraints on the phases are such that:

\section{1. $\mathbf{f}_{\mathbf{k}} \cdot \nabla \times \mathbf{f}_{\mathbf{k}}=0, \forall \mathbf{k}$ (forcing A)}

2. $H_{r}(\mathbf{f})=1$ (forcing B).

The first kind of forcing means that the helicity of the flow is zero at all scales (and in particular $H(\mathbf{f})=0$ ) and the latter expresses that the helicity is maximal for a fixed and given forcing spectrum. Note that similar forcings has been used by Brandenburg [9] in a compressible flow.

\subsection{Comparative study of the flow for both forcings}

The following two simulations are in a 3D periodic box which has a size of $L=2 \pi$. They are based on usual pseudo-spectral method with dealiasing. Grid size is $144^{3}$ : it means that nonlinearities are computed by mean of $144^{3}$ grids to avoid aliasing and linearities by mean of $96^{3}$ grids. Validation of the code has been done, noteworthy, by comparisons with other direct numerical simulations using a different numerical scheme [11]. Initial conditions are $\mathbf{u}=0$ and $\mathbf{b} \sim 0$, i.e. we introduce a weak small-scale magnetic field to avoid a null magnetic field for any time. kinematic viscosity $v$ and magnetic diffusivity are both set equal to 0.005 . In the two simulations, forcing $A$ and forcing $B$ have exactly the same parameter values except for the random selection of the forcing phases (as explained above). 
In Fig. 1, it is shown the time evolution of magnetic and kinetic energies for both forcings. The simulation times $T_{\mathrm{S}} \sim 180$ are not large-enough to study the statistically stationary states. Clearly, in forcing $B$ simulation, the magnetic energy is still slowly growing in mean. In fact, we want to focus, not on the mechanisms allowing the persistence of a large-scale magnetic field, but on the
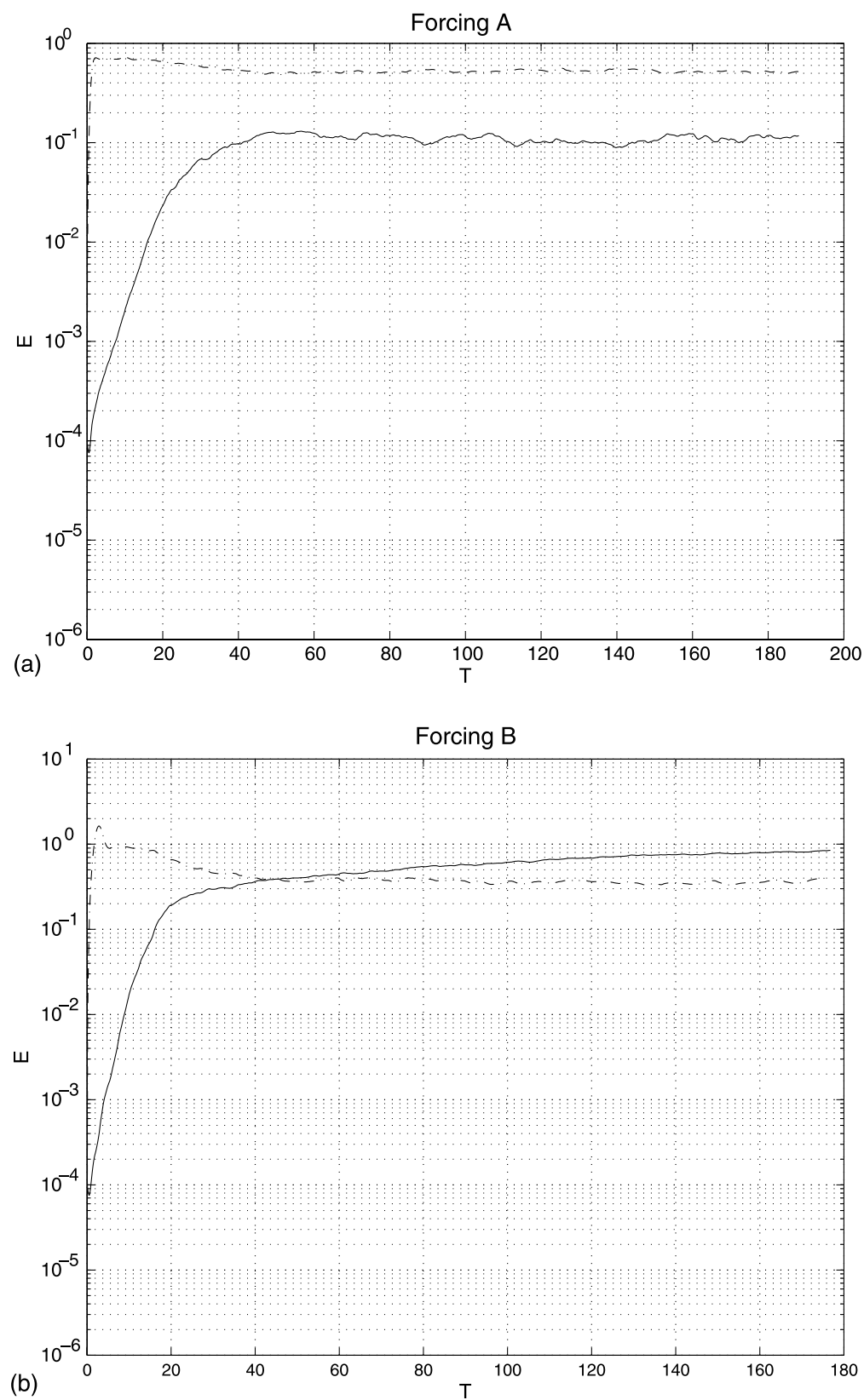

Fig. 1. Time evolution of kinetic energy $E(\mathbf{u})$ (dashed lines), magnetic energy $E(\mathbf{b})$ (solid lines). 
ones linked to its generation. In both cases, as expected, kinetic energy is initially rapidly accumulated. In the second phase, the growth of the magnetic field initiates a decrease of the kinetic energy. Finally, in both simulations, kinetic energy reaches a limit value that is about $40 \%$ lower for the flow with a non-helical forcing. Moreover this last flow reaches also a magnetic stationary state but magnetic energy is small compared to the kinetic one (one fifth) and therefore no important transfer of kinetic energy to magnetic energy occurs. On the contrary, in the helically forced flow, magnetic energy strongly exceeds the kinetic one. This is a dynamo effect signature. Besides, the total energy of the helically forced flow clearly exceeds the forcing $A$ one. It is important to underline the effect of the Lorentz force on the magnetic field. If we suppress it, the velocity field becomes independent of the magnetic field and the kinematic equation (6) is a linear function of the magnetic field. While the magnetic field is weak, time evolution of the magnetic energy is similar with or without the Lorentz force, but in the absence of the Lorentz force and after this transition phase, magnetic energy keeps growing (almost linearly with the time) and never saturates. Differentiation of both evolutions occurs after a few time units in this simulation.

In fact, as it can be seen in Fig. 2, helical forcing generates kinetic helicity. Indeed, with a nonhelical forcing, the relative helicity fluctuates around zero to a few percents, while with the helical forcing and after a transition regime linked to the initial conditions and the forcing $(t \lesssim 20)$, the mean value is about $43 \%$. Note also that helicity saturates to a value five times higher with the helical forcing. As a consequence, in simulation forcing $B$ a helical flow grows (this is not limited to the forcing) and therefore, as already mentioned, this is a situation favorable to an alpha effect. However, for this parameters regime, magnetic helicity is much weaker than kinetic one. Therefore, according to quasi-linear theory [7], back reaction of Lorentz force might not be important enough to promote a strong suppression of the alpha effect. Of course, this last point can become false in more turbulent regimes but a systematic study of an evidence of alpha effect suppression at height $R_{\mathrm{m}}$ is out of the scope of this paper which focus mainly on the definition of the alpha effect.

Fig. 3 shows that turbulent helical flows (forcing B) generate large-scale magnetic fields in agreement with theory and previous works [9]. Indeed, clearly, a pile up of magnetic energy to the largest scales is observed. Note also that the kinetic spectrum is almost stationary. In the nonhelical flow, even at large times $T=160$ (i.e. times large with the characteristic time of energy saturation, around 40 in Fig. 1), there is no tendency to any accumulation of magnetic energy at such a scale (as it could be deduced from a first inspection of the figure) but magnetic energy oscillates around the value $4 \times 10^{-4}$. At small scales, no equipartition of magnetic and kinetic energies (not even a weak deviation) is observed but the spectra follow an exponential law in the dissipation range [5]. While this law is, as expected, still valid in the simulation with forcing $\mathrm{B}$, the tendency to equipartition is much more pronounced. More precisely, this is clearly the case for $k>8$. However, for $1.5 \leqslant k \leqslant 8$ there is also a tendency to an alignment of magnetic and kinetic energies but on large time scales (compare to the simulation time $T_{\mathrm{S}}$ ). In fact, we will verify below that an alpha mechanism develops on time scales lower than $T_{\mathrm{S}}$. Moreover for $k>8$ magnetic energy is always more important than the kinetic one. This has been already observed by Brandenburg [9] and corresponds to theoretical predictions [1]. Note that this behavior is characteristic of scales smaller than the forcing scales.

It is enlightening to visualize snapshots of the dynamics. In Fig. 4, contour plots along $y-z$ plane of the magnetic field for both simulations (performed at a same time $T=160$ for 

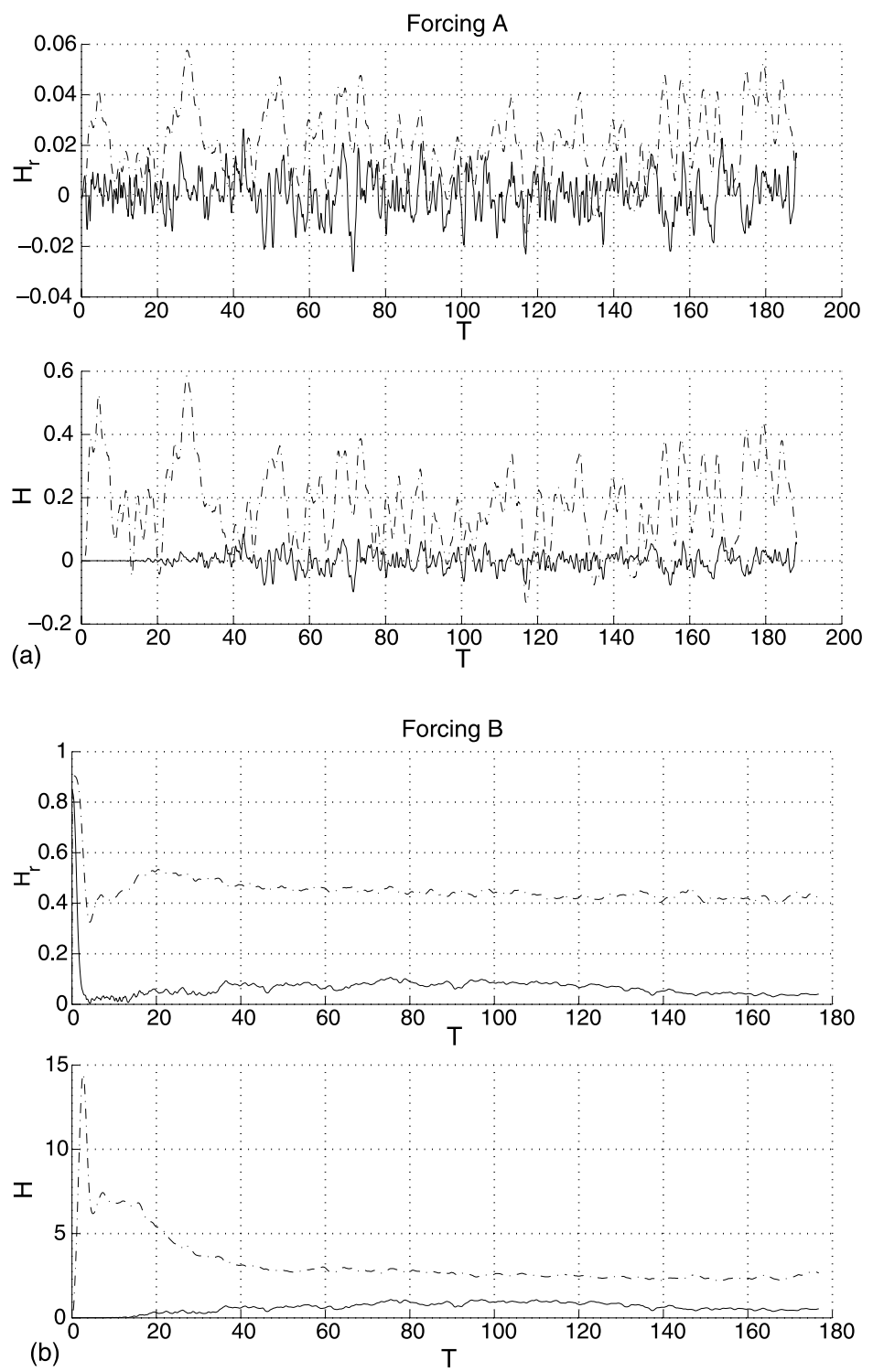

Fig. 2. Time evolution of kinetic (dashed lines) and magnetic (solid lines) relative helicities (up) and helicities (down).

comparisons) indicate a deep difference in the dynamics. Indeed, in the helical flow, conjugate modes $\mathbf{k}=(0,0, \pm 1)$ (i.e. $\left.k_{x}=0, k_{y}=0, k_{z}= \pm 1\right)$ are dominant in the spatial dependence of $b_{x}$ and $b_{y}$ with a phase shift of $\pi / 2$. This is not true for component $b_{z}$. In fact contour plot along some other planes indicate that this is not a plane dependent observation. So following Brandenburg [9], we could say that this behavior could be mathematically written $\mathbf{b}_{\mathrm{LS}}(x, y, z, t) \sim$ $\left(b_{x}^{0}(t) \sin (z+\phi), b_{y}^{0}(t) \cos (z+\phi), 0\right)$. Moreover, we checked that $b_{x}^{0}(t) \sim b_{y}^{0}(t)$ on large time intervals and therefore, in a first approximation, it is obtained that Eq. (1) describes the large-scale dynamics with $\beta$ set equal to zero. Here we call large-scale fields ( $\mathbf{b}_{\mathrm{LS}}$ and $\mathbf{u}_{\mathrm{LS}}$ ) the fields truncated 

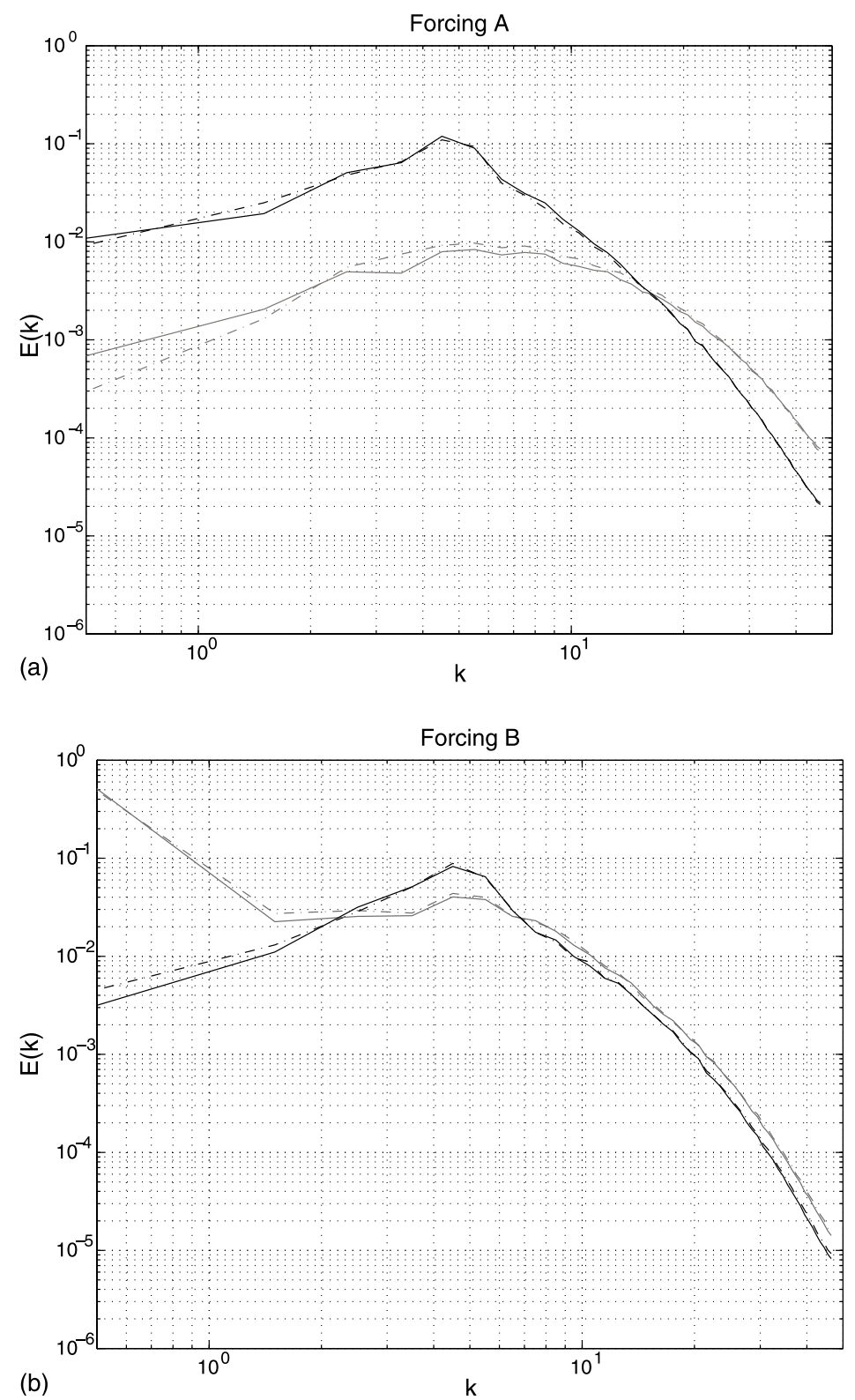

Fig. 3. Spectra of kinetic (black lines) and magnetic (gray lines) energies at times $T=160$ (solid lines) and $T=150$ (dashed lines).

to modes $|\mathbf{k}|=1$. This is natural in this system because there is a pile up of magnetic energy at this scale. It should be noted, however, that there is no strict spatial scale separation as required by standard theory [3]. Indeed there is no intermediate scale $a$ between the size of the system (the length of the box $L$ ) and the typical forcing scale where eddies are generated $l_{f} \sim L / 5$ satisfying $l_{f} \ll a \ll L$. 

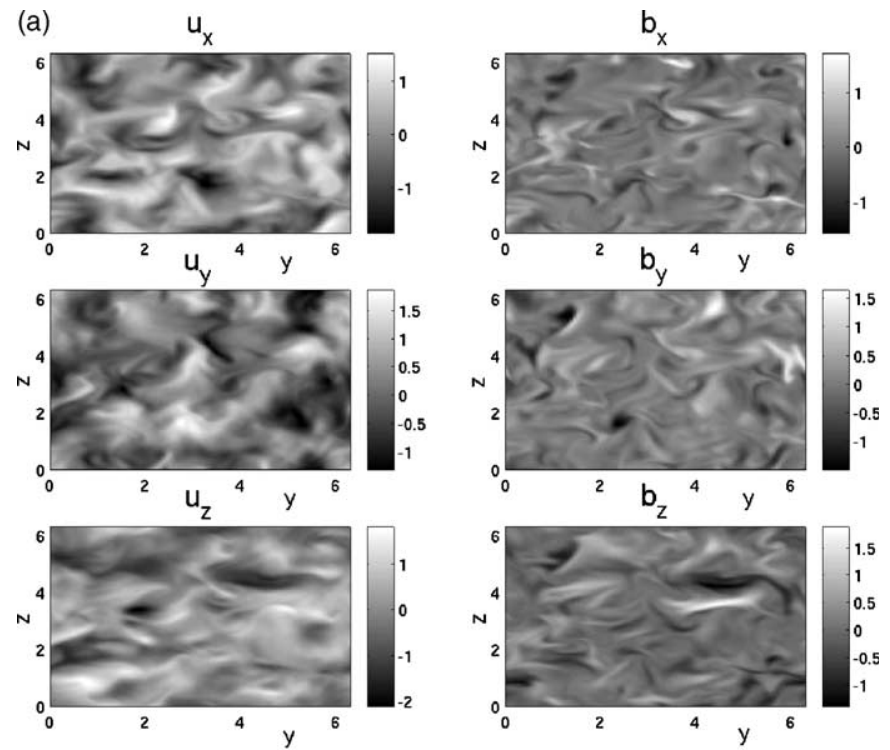

(b)

$u_{x}$
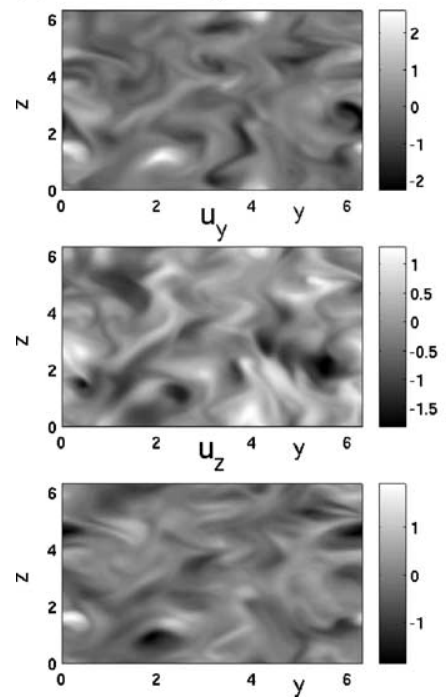

$b_{x}$
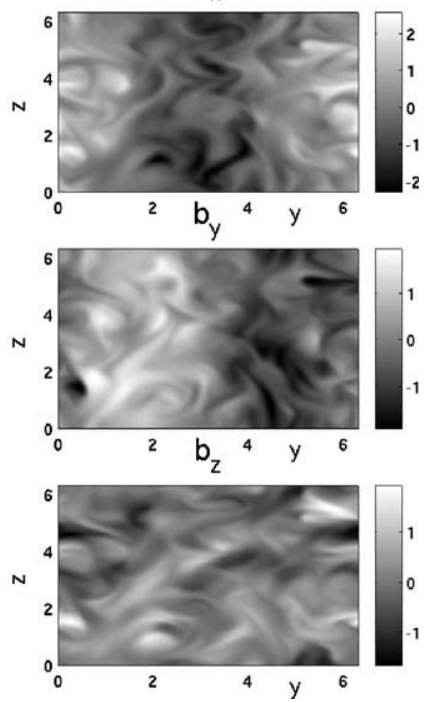

Fig. 4. Snapshot of fields $\mathbf{u}$ and $\mathbf{b}$ in a $y-z$ plane: forcing $A$ (up) and forcing $B$ (down) at time $T=160$.

In the non-helical flow, modes $k=1$ contain a weak fraction of the magnetic energy and no large scale is visible from the snapshots. Indeed, as it can be inspected from the contour plots, most of the volume has a very weak magnetic activity, essentially fluctuations around a null magnetic field. Nevertheless, localized structures on intermediate spatial scales, i.e. with a largest dimension typically between one third and one tenth of the size box, appear. They are generated, and also localized during an important part of their lifetime, in regions of strong velocity gradients (see Fig. 4). Intermittent magnetic structures have been already observed [12]. However, it 
seems that, in simulation forcing $A$, the structure are much more spatially localized: the norm of the magnetic field $b$ (respectively velocity field) is higher than $50 \%$ of $\max (\|\mathbf{b}\|)$ (respectively $\max (\|\mathbf{u}\|)$ ) in only about $1 \%$ (respectively $15 \%$ ) of the total volume. Modeling and also statistical study of such structures does not seem to be done yet. Besides, it would be interesting to search if there is no link to some negative beta effect, i.e. negative magnetic eddy diffusivity. In fact, by mean of a kinematic approach, it has been shown that, in stationary non-helical flow, it can drive instabilities and this mechanism is quite common (see for instance $[13,14]$ ). However the usual beta effect (as described in Eq. (1)) can not be associated to those structures because it implies the existence of unstables modes at the largest scales. More precisely, Lanotte et al. [14] obtained an enhanced small scales beta kinematic dynamo (involving a growth rate proportional to $k^{2}$ ) for a specific stationary velocity flow, i.e. they obtained a primary dynamo mechanism not acting on large-scale magnetic structures. The importance of this kind of dynamo in non-kinematic and time dependent flows is not clear but the localized intermittent structures observed in simulation forcing $A$ might be associated to it.

\subsection{Helical waves and non-local alpha effect}

In order to study in detail the large-scale behavior of the flow, in Fig. 5 we plot the time evolution of all the amplitudes of the largest modes of the magnetic field $\mathbf{A}^{\mathbf{k}}(t)$ (with $|\mathbf{k}|=1$ ). In
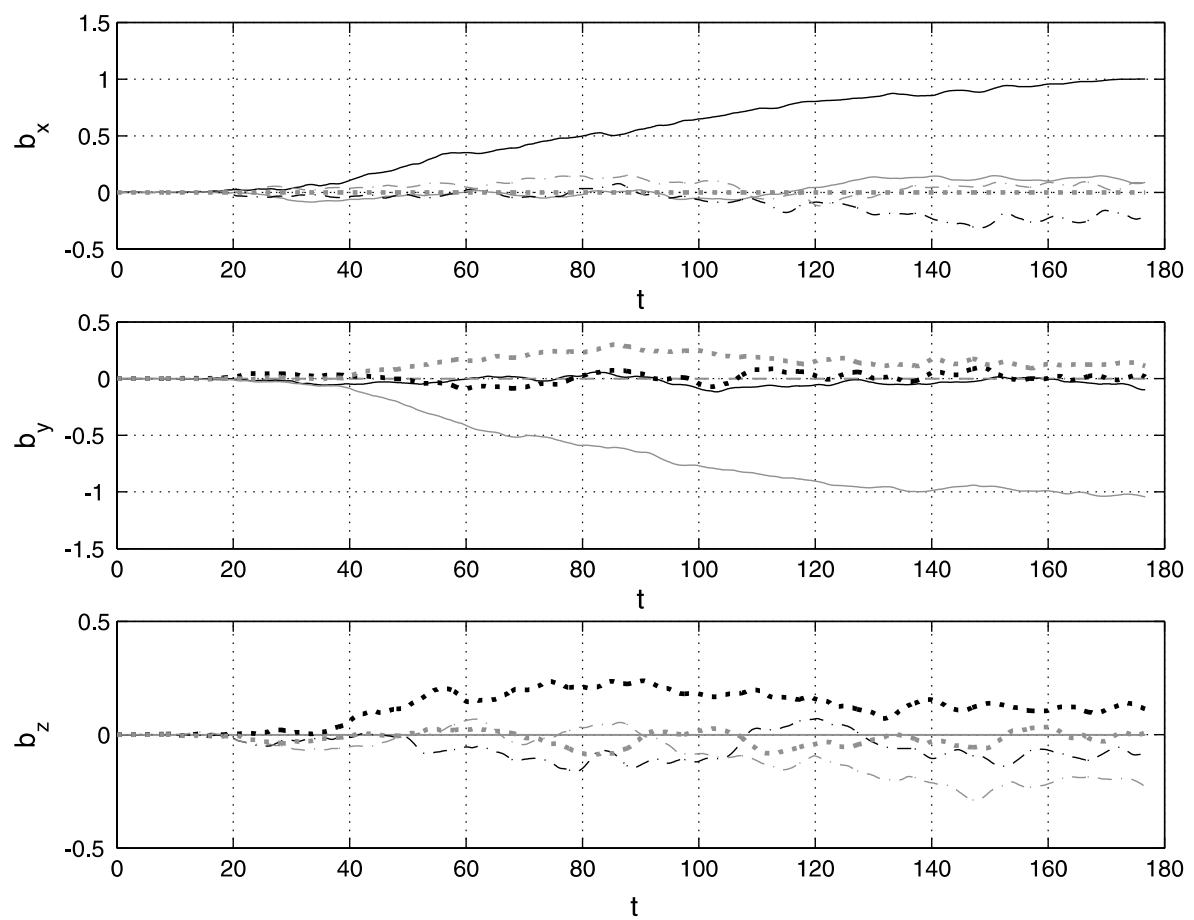

Fig. 5. Forcing $B$ simulation - time evolution of magnetic large-scale mode vector amplitudes $\mathbf{A}^{\mathbf{k}}$ : Real $\mathbf{A}^{(0,0,1)}$ (black, solid), Imag $\mathbf{A}^{(0,0,1)}$ (gray, solid), Real $\mathbf{A}^{(0,1,0)}$ (black, dashdot), Imag $\mathbf{A}^{(0,1,0)}$ (gray, dashdot), Real $\mathbf{A}^{(1,0,0)}$ (black, dotted), Imag $\mathbf{A}^{(1,0,0)}$ (gray, dotted). 
the following, we restrict ourselves to modes such that $k_{x}+k_{y}+k_{z}=1$ because of the real nature of the magnetic field $\left(\mathbf{A}^{-\mathbf{k}}=\left(\mathbf{A}^{\mathbf{k}}\right)^{*}\right)$. It is clear that the components of the modes are not independent of each other. For instance the real part of the amplitude $A_{x}^{(0,0,1)}$ (black solid line in the top figure) and the imaginary part of $A_{y}^{(0,0,1)}$ (gray dashdot line in the middle figure) are linked by the relation Real $A_{x}^{(0,0,1)} \sim-\operatorname{Imag} A_{y}^{(0,0,1)}$, while $A_{z}^{(0,0,1)} \sim 0$. In fact this suggests that time evolution of the three large-scale magnetic modes are obeying independently to an alpha process. At this point it is useful to introduce the notion of complementary amplitudes. Suppose that a given mode $\mathbf{b}_{\mathbf{k}}(\mathbf{x}, t)=\mathbf{A}^{\mathbf{k}}(t) \exp (\mathbf{i k} \cdot \mathbf{x})$ is driven by an alpha process

$$
\frac{\partial}{\partial t} \mathbf{b}_{\mathbf{k}}(\mathbf{x}, t)=\alpha_{\mathbf{k}}(t) \nabla \times \mathbf{b}_{\mathbf{k}}(\mathbf{x}, t)
$$

and that initially the amplitude of the mode is zero (in agreement with our initial conditions). It is straightforward to obtain that, first $\mathbf{A}^{\mathbf{k}}$ is orthogonal to $\mathbf{k}$ at all times, and second, if we introduce $\left(a_{i}(t)\right)_{i=1,2,3}$ the complex components of $\mathbf{A}^{\mathbf{k}}$ in an orthonormal basis with $a_{3}=\mathbf{A}^{\mathbf{k}} \cdot \mathbf{k} / k$, then $a_{1}= \pm \mathbf{i} a_{2}, a_{3}=0$. We call the couple $\left(a_{1}, a_{2}\right)$ the complex complementary amplitudes of mode $\mathbf{k}$. As a consequence

$$
\begin{aligned}
& \text { Real } a_{1}=\mp \operatorname{Imag} a_{2}, \\
& \operatorname{Imag} a_{1}= \pm \operatorname{Real} a_{2}, \\
& a_{3}=0 .
\end{aligned}
$$

We now check if the large-scale modes evolution is in agreement with Eq. (8). First, it is easily seen in Fig. 5 that Eq. (11) is true for the three modes. Second, we plot in Fig. 6 the real and imaginary part of the complementary amplitudes. More precisely, for each of the three large-scale modes, we plot with the same line type (but a different color) Real $a_{1}$ and $\mp \operatorname{Imag} a_{2}$ (with the right sign $\mp$ in order to get the superposition of the curves if any), and also Real $a_{2}$ and $\mp \operatorname{Imag} a_{1}$. Therefore there are four plots and two line types for each of the three modes. This graph shows clearly that Eqs. (9) and (10) describe the large-scale dynamics with a good precision and this is not limited to the mode containing the largest amount of magnetic energy $(\mathbf{k}=(0,0,1)$ in this simulation. As a consequence, we obtain the main numerical result of this paper, that is, Eq. (8) (and not (1), with a null beta) is the appropriate model to describe large-scale dynamics of the magnetic field in helical turbulence. Besides, we can write a dynamic equation for the large-scale magnetic field $\mathbf{b}_{\mathrm{LS}}(\mathbf{x}, t)=\sum_{|\mathbf{k}|=1} \mathbf{b}_{\mathbf{k}}(\mathbf{x}, t)$ which is

$$
\frac{\partial}{\partial t} \mathbf{b}_{\mathrm{LS}}(\mathbf{x}, t)=\alpha_{\mathrm{LS}} * \nabla \times \mathbf{b}_{\mathrm{LS}}(\mathbf{x}, t),
$$

where the symbol $*$ denotes a convolution product. $\alpha_{\mathrm{LS}}$ is the inverse Fourier transform of the $\alpha_{\mathbf{k}}(t)$ defined in Eq. (8) where all non-large-scale Fourier components are zero. Let us note, however, that the usual model equation is recovered from (12) when one mode $\mathbf{k}_{d}$ is strongly dominant from an energetic point of view, i.e. when $\alpha_{\mathbf{k}_{d}} \gg \alpha_{\mathbf{k}}, \forall \mathbf{k} \neq \mathbf{k}_{d}$. In fact, this last point explains the partial validity of the model equation (1) in the simulation because one mode is dominant. However, it is important to note that the coexistence of a bath of helical waves at large scales is not simple to explain because their mutual interactions via Lorentz force is not weak à priori: first, because this is a non-linear magnetic regime (as already explained), second because 


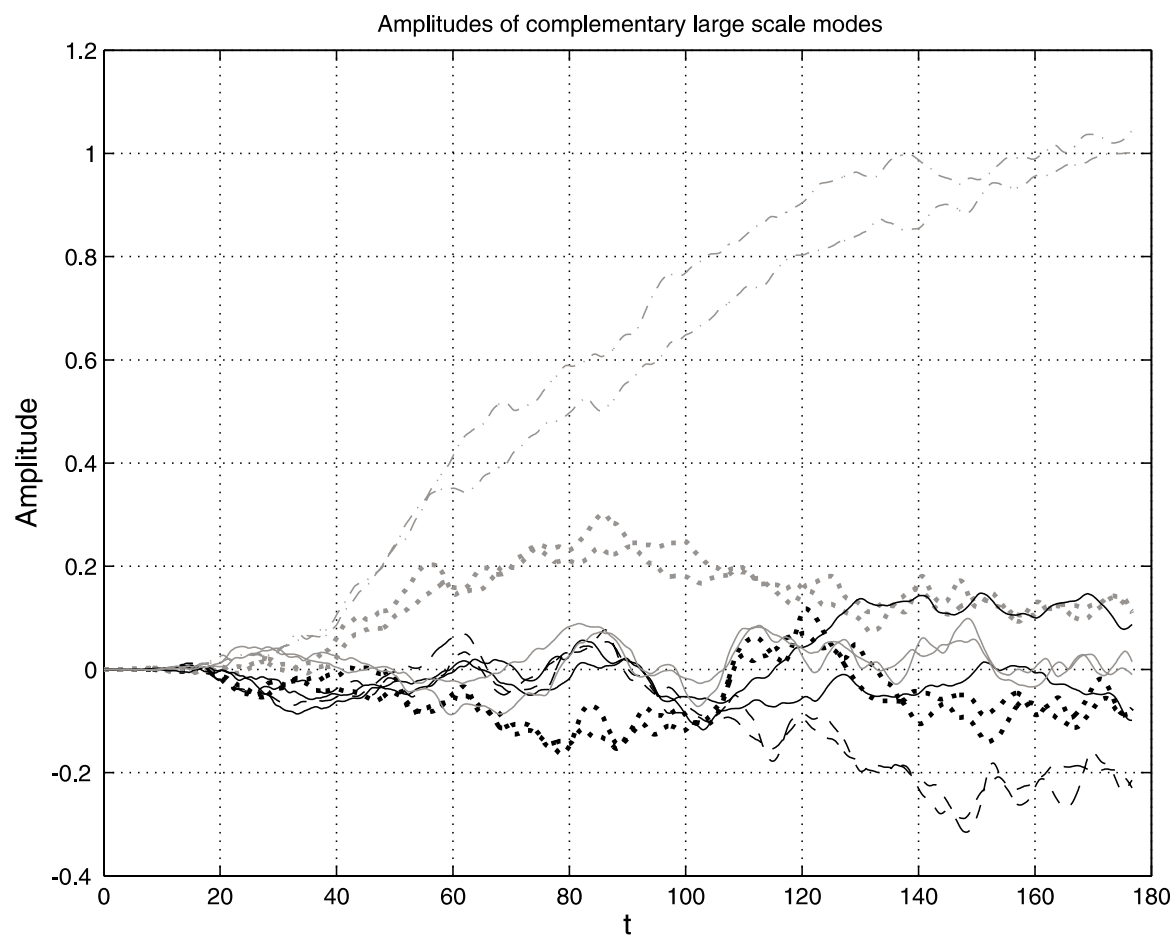

Fig. 6. Comparison of pairs of real complementary amplitudes: $\mathbf{k}=(0,0,1)$ (gray dashdot lines and black solid lines), $\mathbf{k}=(0,1,0)$ (black dashed lines and black dotted lines) and $\mathbf{k}=(1,0,0)$ (gray dotted lines and gray solid lines).

they concentrate a large fraction of the total energy. One may think for instance that the interaction of two helical waves can lead to the suppression of the helical character of those waves.

It is interesting to focus on deviations from the model equation (8). First, the distance between the largest complementary amplitudes (mode $\mathbf{k}=(0,0,1)$, gray dashdot lines in the figure) slightly increases around times 60 and 95 and decreases again at time 150. In between those fast processes, the gap is essentially constant in agreement with (8). One could think that this is correlated to the value of the amplitudes and therefore to the non-linearities of the MHD equations, but this does not explain the decrease of the distance at time 150. Second, the two others real complementary modes of mode $\mathbf{k}=(0,0,1)$ in between times 105 and 125 also show a strong increase of their mutual distance (black solid lines). Besides, one of this two modes seems to be linked with two others complementary modes (mode $\mathbf{k}=(1,0,0)$, blue lines) at larger times. Detailed analysis of these phenomena (observed in most of the simulations we performed) is out of the scope of this paper. It would be also interesting to obtain situations where amplitudes of two or more largescale modes are larger than 1 in order to test the model in stronger large-scale non-linear regimes.

Pouquet et al. [1] proposed a dynamo mechanism where the inverse cascade results from a competition between the helicity and Alfvén effects. More precisely, let us call $k$ the mode for which we want to determine the mechanisms linked to the variation of the magnetic energy $E_{k}^{\mathrm{M}}(t)$ and of the helicity $H_{k}(t)$. They mainly distinguished two kinds of non-local effects involving interacting triads $(k, p, q)$ : $\left(\mathrm{i}_{k}\right) k \ll p \sim q$ which is linked to alpha or helicity effect, and, (ii $\left.{ }_{k}\right)$ 
$p \ll k \sim q$ which is linked to Alfvén effect. By focusing on the largest scales in the study above, we naturally eliminated (ii ${ }_{k}$ ). We obtained that a convolution (cf Eq. (12)) is involved in the alpha mechanism $\left(\mathrm{i}_{k}\right)$ and it is therefore natural to propose the conjecture that this is true at the scales larger than the forcing scales and around the one where magnetic energy $E_{k}^{\mathrm{M}}(t)$ is maximum. Indeed, $E_{k^{\prime}<k}^{\mathrm{M}}(t)$ decreases strongly when $k^{\prime}$ decreases, inhibiting a strong Alfvén effect. In this context, $\alpha_{\mathbf{k}}$ is different from zero in a $k$-space narrow interval $I(t)$ centered around a value $k_{\text {peak }}(t)$ such that $\max _{k}\left(E_{k}^{\mathrm{M}}\right)=E_{k_{\text {peal }}}^{\mathrm{M}}$, and such that $I(t)=\{k=1\}$ when $t$ is large enough. It is straightforward to obtain from (12) that $\mathrm{d} E_{k}^{\mathrm{M}} / \mathrm{d} t=\left\langle k^{\prime 2} \alpha_{\mathbf{k}^{\prime}} \mathbf{a}_{\mathbf{k}^{\prime}} \cdot \mathbf{b}_{\mathbf{k}^{\prime}}\right\rangle_{I}$ where the brackets denotes a mean over the modes $\mathbf{k}^{\prime}$ included in the interval $I$, and $\mathbf{a}$ is the potential vector. This is of course in agreement with $\mathrm{d} E_{k}^{\mathrm{M}} / \mathrm{d} t \sim k^{2} \alpha_{k} H_{k}$, the formula obtained by Pouquet et al. [1], and indicates that this last formula is linked to the non-linear coexistence of helical waves at scales $\sim k_{\text {peak }}$.

\section{Conclusion}

In this paper, we have presented a comparative study of helical and non-helical turbulence in the frame of resistive MHD turbulence. We obtained, in agreement with previous works, that an helical forcing generates a dynamo, the dominant mechanism of which is an alpha effect. In the literature, alpha effect is usually associated up to some minor modifications and with some gradations, to the model equation (1). For instance, Cattaneo et al. [6] treat this equation as "only a convenient way to express the relative importance of the production terms". As another example, Brandenburg [9] devoted more attention to this model and computed alpha under the hypothesis that this is a scalar parameter slowly evolving in time. We verified that our results agree with his in a first approximation. The main result of this paper is that in helical turbulence, alpha effect is a multi-modal non-linear mechanism and can be associated to a simple paradigm equation (12). The validity of this model equation from a non-kinematic point of view, i.e. if we do not neglect the back reaction of the magnetic field on $\alpha_{\mathrm{LS}}$ and, thus, introduce non-linearities generated by the magnetic field, is not trivial. Besides, this equation involves a convolution operator which characterizes its non-local nature. It is noteworthy that, first, these results are to some extends in agreement with the theoretical works of Pouquet et al. [1] and, second, Eq. (12) is much more than a convenient model equation, at least in the regimes studied. Moreover it does not seem necessary to force at very small scales (and to introduce some two scales turbulence) for this model to be valid. Another interesting observation issued from this work is that non-helical turbulence is dominated by intermittent and localized magnetic structures, still to be studied in detail, evolving in a practically non-magnetized environment.

\section{Acknowledgements}

This work was started during the "Festival de Théorie", International Workshop on Self Organization and Transport in Electromagnetic Turbulence, Aix-en-Provence, France, 2001. It is a pleasure to thank P. Diamond and J. Paulsen for stimulating discussions, and also J. Léorat for interesting comments on this work. 


\section{References}

[1] Pouquet A, Sulem PL, Léorat J. Strong mhd helical turbulence and the nonlinear dynamo effect. J Fluid Mech 1976;77:321-54.

[2] Moffat K. Encyclopedia of astronomy and astrophysics. Bristol UK: Nature Publishing Group; 2001 [Chapter: Dynamo Theory].

[3] Moffat K. Magnetic field generation in electrically conducting fluids. Cambridge: Cambridge University Press; 1978.

[4] Krause F, Rädler KH. Mean field magnetohydrodynamics and dynamo theory. Berlin: Akademie; 1980.

[5] Biskamp D. Nonlinear magnetohydrodynamics. Cambridge: Cambridge University Press; 1993.

[6] Cattaneo F, Hughes DW. Nonlinear saturation of the turbulent $\alpha$ effect. Phys Rev E 1996;54:4532.

[7] Gruzinov AV, Diamond PH. Nonlinear mean field electrodynamics of turbulent dynamos. Phys Plasmas 1996;3:1853.

[8] Blackman EG, Field GB. Constraints on the magnitude of $\alpha$ in dynamo theory. Astrophys J 2000;534:984.

[9] Branderburg A. The inverse cascade and nonlinear alpha-effect in simulations of isotropic helical hydromagnetic turbulence. Astrophys J 2001:824.

[10] Branderburg A, Donner KJ. MNRAS 1997;288:L29.

[11] Agullo O, Müller W-C, Knaepen B, Carati D. Large eddy simulation of decaying magnetohydrodynamic turbulence with dynamic subgrid-modelling. Phys Plasmas 2001;8(7):3502.

[12] Meneguzzi M, Frisch U, Pouquet A. Helical and nonhelical turbulent dynamos. Phys Rev Lett 1981;47:1060.

[13] Zheligovsky VA, Podviginia OM, Frisch U. Dynamo effect in parity-invariant flow with large and moderate separation of scales. Geophys Astrophys Fluid Dynam 2001;95:227-68.

[14] Lanotte A, Noullez A, Vergassola M, Wirth A. Large-scale dynamo produced by negative eddy diffusivities. Geophys Astrophys Fluid Dynam 1999;91:131-46. 